\title{
Tratamento endovascular de aneurisma de ilíaca roto: relato de caso
}

\author{
Endovascular treatment of a ruptured iliac aneurysm: case report \\ Adinaldo Adhemar Menezes da Silva1, Luis Fernando Reis' ${ }^{1}$, Daniel Gustavo Miquelin², \\ Priscilla Yukiko Sano ${ }^{2}$, Vlanna Sales Pereira ${ }^{3}$, José Maria Pereirade Godoy ${ }^{4}$
}

\section{Resumo}

A taxa de mortalidade cirúrgica do aneurisma de ilíaca roto é similar à do aneurisma de aorta abdominal roto, devido à sua localização profunda na pelve, dificuldade de exposição distal da ilíaca decorrente do hematoma, bridas devido a laparotomia prévia e proximidade com ureter e estruturas venosas. O objetivo do presente estudo é enfatizar o procedimento endovascular como mais uma opção na correção dessas lesões. Relata-se o caso de um paciente de 60 anos de idade, submetido a derivação com enxerto aorto-biilíaco prévio com prótese há 5 anos, por aneurisma de aorta abdominal infra-renal, apresentando rotura de aneurisma em segmento remanescente da ilíaca comum esquerda. Estava hemodinamicamente estável após ressuscitação com fluidos e foi submetido ao tratamento endovascular de urgência, com a exclusão do aneurisma e ausência de vazamentos.

Palavras-chave: Aneurisma de ilíaca roto, tratamento endovascular de aneurisma, endoprótese.

\section{Introdução}

Os aneurismas de artérias ilíacas (AAI) são raros e representam 2 a $7 \%$ dos aneurismas do segmento aorto-ilíaco $^{1,2}$.

$\mathrm{O}$ aparecimento de AAI após reparo do aneurisma de aorta abdominal (AAA) é variável $(0,6-1,2 \%)$, subestimado, devido à dificuldade de detecção clínica, e relacionado com o tempo de seguimento pós-operatório. A maioria das séries relata nova formação ou

1. Professor, Serviço de Angiologia e Cirurgia Vascular, Faculdade de Medicina de São José do Rio Preto (FAMERP), São José do Rio Preto, SP.

2. Médico, Serviço de Angiologia e Cirurgia Vascular, FAMERP, São José do Rio Preto, SP.

3. Residentes, Serviço de Angiologia e Cirurgia Vascular, FAMERP, São José do Rio Preto, SP.

4. Professor livre-docente, Serviço de Angiologia e Cirurgia Vascular, FAMERP, São José do Rio Preto, SP.

Artigo submetido em 13.05.06, aceito em 01.01.07.

J Vasc Bras 2007;6(1):78-81.

Copyright $\odot 2007$ by Sociedade Brasileira de Angiologia e de Cirurgia Vascular.

\begin{abstract}
The surgical mortality rate of ruptured iliac aneurysms is similar to ruptured abdominal aortic aneurysms in terms of their location deep in the pelvis, difficult access to the distal iliac artery due to hematoma, adherences due to prior laparotomy and proximity of the ureter and venous structures. This study aims at highlighting the endovascular procedure as an alternative in the correction of this lesion. We report the case of a patient submitted to aortobiiliac prosthetic graft due to infrarenal abdominal aortic aneurysm 5 years ago, who presented with a ruptured aneurysm in the remaining segment of the left common iliac artery. The patient was hemodynamically stable after fluid replacement therapy and was submitted to urgent endovascular treatment. The treatment resulted in the exclusion of the aneurysm without endoleaks.
\end{abstract}

Keywords: Ruptured iliac aneurysm, endovascular treatment of aneurysms, endograft.

progressão da doença aneurismática em torno de 4 a 4,5 anos, sendo acima de 5 anos na maioria dos casos ${ }^{3}$.

Os pacientes portadores de AAI são freqüentemente assintomáticos (em torno de 50\%) e, em geral, observados nos exames de imagem para avaliação de outras condições clínicas, como disfunções urológicas, ginecológicas e gastrointestinais. Clinicamente, podem se apresentar com sintomas relacionados à compressão de estruturas adjacentes, gerando sintomas inespecíficos e comuns a outras doenças. A complicação mais temida e muitas vezes a única forma de apresentação da doença - é a ruptura do saco aneurismático. A freqüência dessa complicação é variável, e recomenda-se o reparo eletivo desses aneurismas quando atingem $3 \mathrm{~cm}$, na ausência de outras doenças graves ou risco cirúrgico elevado ${ }^{3}$.

A taxa de mortalidade associada ao tratamento cirúrgico dos AAI rotos situa-se em torno de 33\%, semelhante à dos AAA rotos. Os AAI apresentam particularidades técnicas na sua abordagem cirúrgica, 
que os tornam mais difíceis para o reparo que os AAA: apresentam localização profunda na pelve, proximidade com o ureter, relação íntima com veias e exposição difícil dos ramos distais, principalmente na presença de ruptura com sangramento e bridas devido a laparotomia prévia $^{1-3}$.

O tratamento endovascular de urgência nos aneurismas aorto-ilíacos rotos é uma opção terapêutica sob avaliação e tem mostrado resultados satisfatórios. Esses achados podem estar relacionados à condição menos invasiva do método, por se evitar a laparotomia, diminuindo o sangramento intra-operatório e a utilização de anestesia local ${ }^{4-6}$.

\section{Relato de caso}

Paciente, 60 anos, masculino, branco e sem acompanhamento anterior no serviço. Apresentou quadro de dor abdominal súbita em mesogástrio e quadrante inferior esquerdo (4 horas de evolução), associada a sinais de choque hipovolêmico. Ultra-som de abdome, realizado no serviço de origem e encaminhado com o doente, mostrava coleção líquida ao redor de prótese vascular utilizada em cirurgia prévia e hipótese de ruptura de enxerto. No setor de emergência, durante sua admissão, infundiu-se $1.000 \mathrm{~mL}$ de solução cristalóide e uma unidade de concentrado de hemácias. $\mathrm{O}$ doente melhorou da instabilidade hemodinâmica, mantendo-se com dor e massa pulsátil à palpação nas regiões mesogástrica e hipogástrica esquerda. Apresentava outros antecedentes, como correção de AAA infrarenal há 5 anos, com prótese aorto-biilíaca comum, e houve dilatação da ilíaca abaixo da anastomose, insuficiência renal crônica em hemodiálise e acidente vascular cerebral isquêmico (AVC) há 2 anos. Tomografia computadorizada (TC) de urgência mostrou enxerto aortobiilíaco patente e aneurisma com sinais de ruptura em segmento remanescente da artéria ilíaca comum esquerda (Figura 1). Devido à presença de comorbidades e fragilidade do estado de saúde, optou-se pelo tratamento endovascular. O paciente foi conduzido ao setor de hemodinâmica, onde a angiografia mostrou presença de AAI comum esquerda com extravasamento do meio de contraste preenchendo toda a cavidade do pseudoaneurisma recém-formado (Figura 2A).

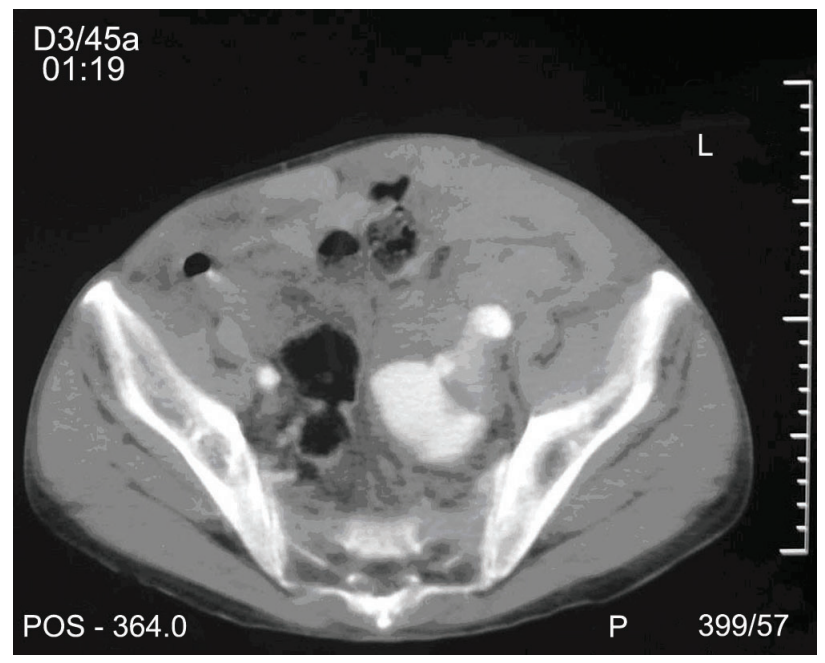

Figura 1 - Tomografia computadorizada evidenciando aneurisma roto em segmento remanescente de artéria ilíaca comum esquerda na bifurcação ilíaca

O paciente iniciou quadro de confusão mental e agitação, requerendo anestesia geral. Acessou-se cirurgicamente a artéria femoral comum esquerda e por arteriotomia transversa e foram implantadas, de forma seqüencial, três endopróteses (uma de 14/12 x $80 \mathrm{~mm}$ e duas de $12 / 12$ × $60 \mathrm{~mm}$ - Braile Biomédica $^{\circledR}$ ). A primeira endoprótese foi ancorada no ramo esquerdo da prótese de dácron previamente existente, que migrou. Uma segunda endoprótese foi colocada entre o ramo esquerdo da prótese de dácron e a primeira endoprótese. O terceiro módulo foi instalado até alcançar bom segmento da artéria ilíaca externa, excluindo o aneurisma por ausência completa de endovazamentos (Figura 2B). O paciente foi encaminhado à unidade de terapia intensiva e, durante sua evolução, apresentou dificuldade para extubação, devido à exacerbação do distúrbio de deglutição já existente e causado pelo AVC, sendo submetido à traqueostomia no $15^{\circ}$ dia pósoperatório (PO) e desmame do respirador com êxito. No $19^{\circ} \mathrm{PO}$, o paciente apresentou melena por hemorragia digestiva alta (HDA) devido a úlcera duodenal (Forrest IIB), e foi iniciado tratamento clínico. Controle tomográfico das endopróteses no $40^{\circ} \mathrm{PO}$ mostrou bom posicionamento e ausência de endovazamentos, com diminuição acentuada do hematoma (Figura 3). No $49^{\circ}$ PO, o paciente evoluiu para o óbito por recidiva da HDA. 

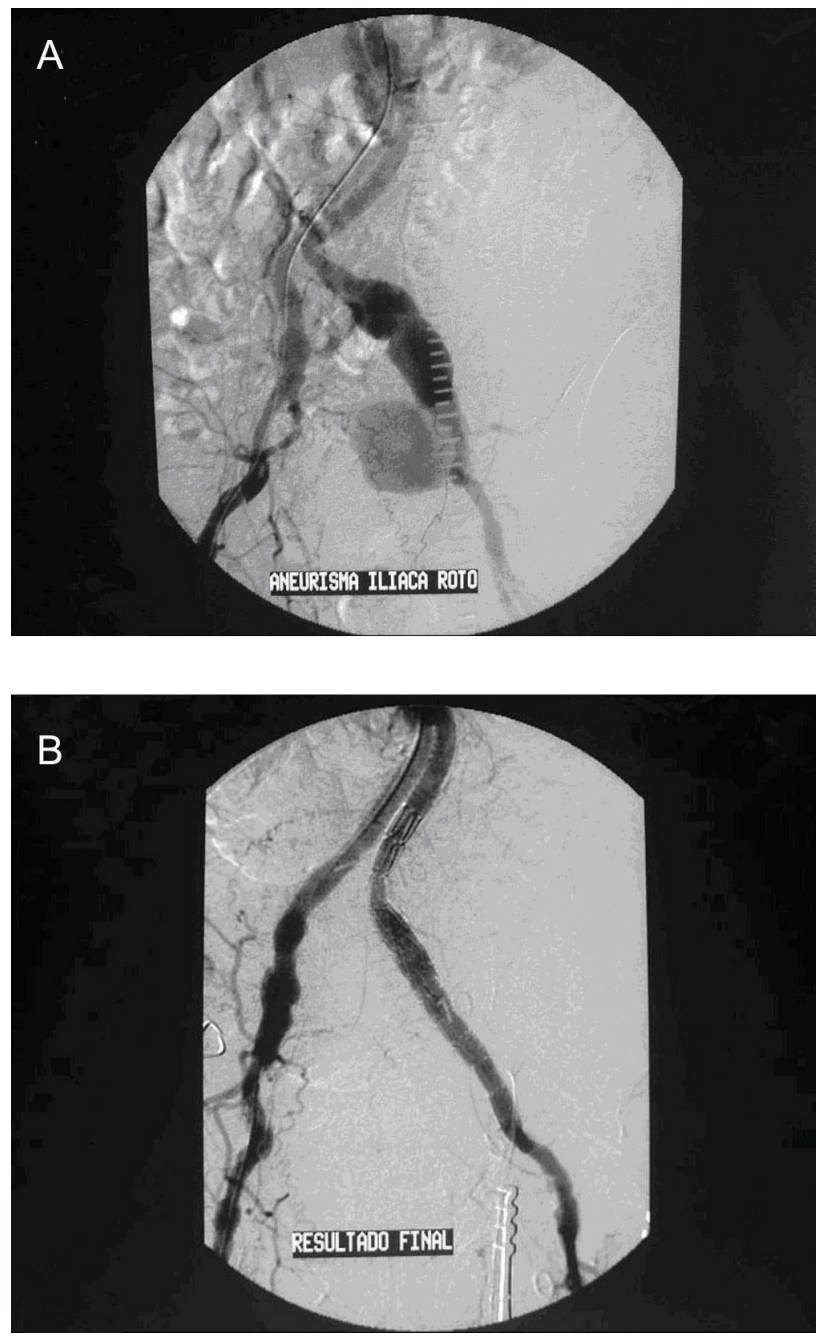

Figura 2 - Aortografia: A) enxerto aorto-biilíaco patente e presença de AAI comum esquerda com extravasamento do meio de contraste preenchendo toda a cavidade do pseudo-aneurisma recém-formado; B) controle angiográfico após implante das endopróteses, ausência de endovazamentos

\section{Discussão}

O presente caso relata a possibilidade de tratamento endovascular do AAI roto em paciente que permaneceu estável hemodinamicamente e com anatomia favorável à colocação da endoprótese. A presença de laparotomia prévia, associada a hematoma pélvico, reforçou essa indicação, já que as condições clínicas presentes conferiam um risco elevado para a cirurgia convencional. Empregou-se anestesia geral, devido ao estado de agitação do paciente, que impossibilitou a anestesia local.

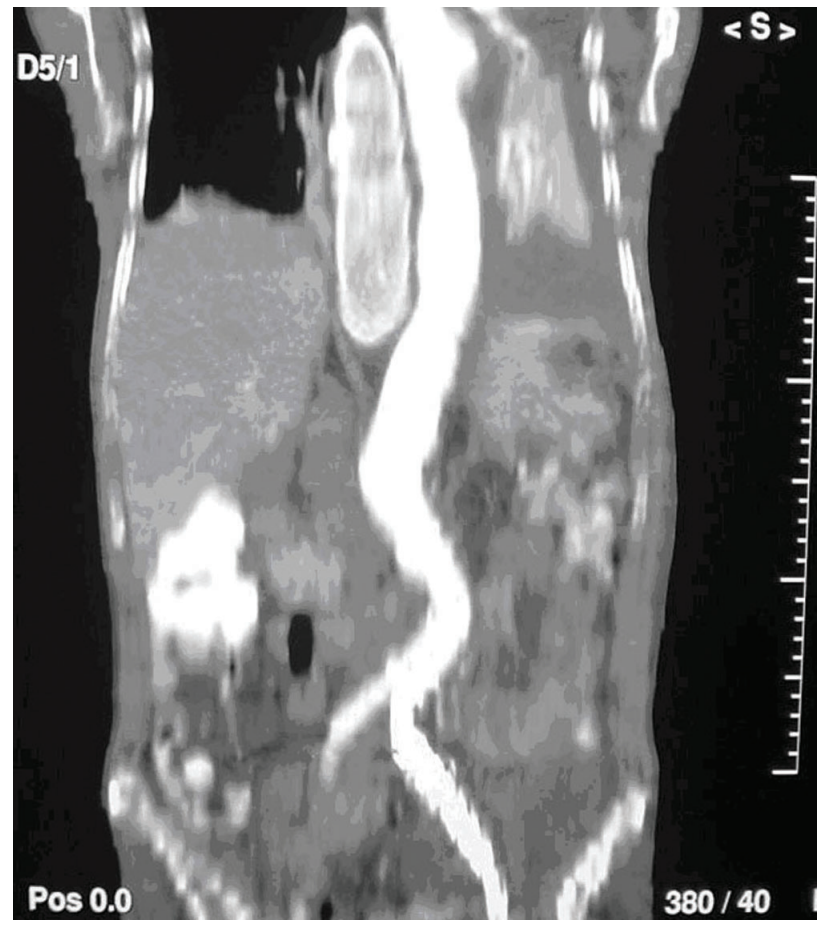

Figura 3 - Tomografia computadorizada realizada no $40^{\circ}$ PO: bom posicionamento das endopróteses, ausência de endovazamentos, redução do hematoma

O tratamento padrão para os aneurismas aortoilíacos rotos é a laparotomia com enxerto aórtico sob anestesia geral ${ }^{4}$. Alguns estudos mostram as vantagens do tratamento endovascular dos aneurismas aortoilíacos rotos em relação à cirurgia convencional, pois evita a laparotomia, pode ser realizado com anestesia local ou loco-regional, diminui a perda sangüínea, mantém a integridade da parede abdominal e reduz a morbidade e a mortalidade decorrentes das lesões iatrogênicas causadas pela exploração do hematoma retroperitoneal e anatomia distorcida. Os pacientes submetidos ao tratamento endovascular de aneurisma roto descritos em outras séries apresentavam estabilidade hemodinâmica após ressuscitação com fluidos para realização de $\mathrm{TC}$, alto risco cirúrgico ou abdome hostil (obesos, cirurgia abdominal prévia, neoplasia abdominal) ${ }^{1,4,5,7-9}$.

Alguns fatores limitam a realização do tratamento endovascular de urgência, como a necessidade de tomografia pré-operatória, dificuldade na obtenção de endopróteses com medidas apropriadas para cada paciente e 
maior tempo para vedação completa do aneurisma, quando comparado ao tempo para o pinçamento aórtico durante a cirurgia. Esse último fator de limitação tem sido reduzido com o uso de balão por punção percutânea da artéria femoral ou axilar, promovendo a oclusão aórtica até o implante da endoprótese ${ }^{4-6}$.

O tratamento endovascular eletivo do aneurisma de ilíaca tem apresentado bons resultados ${ }^{10}$, sendo cada vez mais aceito como opção de tratamento. Acreditamos que essa modalidade de tratamento possa ser empregada em condição de urgência, desde que um fluxograma seja criado, provendo exame tomográfico ágil, equipe médica especializada, recursos materiais apropriados e presentes no setor e avaliação rigorosa do estado hemodinâmico do paciente ${ }^{10}$. $\mathrm{O}$ seguimento desses pacientes é fundamental, visando identificar as intercorrências e interferir quando necessário ${ }^{10}$.

\section{Referências}

1. Ricci MA, Najarian K, Healey CT. Successful endovascular treatment of a ruptured internal iliac aneurysm. J Vasc Surg. 2002;35:1274-6.

2. Marin ML, Veith FJ, Lyon RT, Cynamon J, Sanchez LA. Transfemoral endovascular repair of iliac artery aneurysms. Am J Surg. 1995;170:179-82.

3. Dosluoglu HH, Dryjski ML, Harris LM. Isolated iliac artery aneurysms in patients with or without previous abdominal aortic aneurysm repair. Am J Surg. 1999;178:129-32.
4. Hechelhammer L, Lachat ML, Wildermuth S, Bettex D, Mayer D, Pfammatter T. Midterm outcome of endovascular repair of ruptured abdominal aortic aneurysms. J Vasc Surg. 2005;41:752-7.

5. Ohki T, Veith FJ, Sanchez LA, et al. Endovascular graft repair of ruptured aortoiliac aneurysms. J Am Coll Surg. 1999;189:102-12; discussion 112-3.

6. Ohki T, Veith FJ. Endovascular grafts and other image-guided catheter-based adjuncts to improve the treatment of ruptured aortoiliac aneurysms. Ann Surg. 2000;232:466-79.

7. Bierdrager E, Lohle PN, Schoemaker CM, Lampmann LE, van Berge Henegouwen DP, Hamming JF. Successful emergency stenting of acute ruptured false iliac aneurysm. Cardiovasc Intervent Radiol. 2002;25:72-3.

8. Williamson AE, Annunziata G, Cone LA, Smith J Endovascular repair of a ruptured abdominal aortic and iliac artery aneurysm with an acute iliocaval fistula secondary to lymphoma. Ann Vasc Surg. 2002;16:145-9.

9. Lee WA, Hirneise CM, Tayyarah M, Huber TS, Seeger JM. Impact of endovascular repair on early outcomes of ruptured abdominal aortic aneurysms. J Vasc Surg. 2004;40:211-5.

10. Sahgal A, Veith FJ, Lipsitz E, et al. Diameter changes in isolated iliac artery aneurysms 1 to 6 years after endovascular graft repair. J Vasc Surg. 2001;33:289-94; discussion 294-5.

Correspondência:

José Maria Pereira de Godoy

Rua Floriano Peixoto, 2950

São José do Rio Preto, SP

CEP 15010-020

E-mail: godoyjmp@riopreto.com.br 